\title{
Booms and Busts: the Burstiness of Star Formation in Nearby Dwarf Galaxies
}

\author{
A. A. Cole $\mathrm{A}$ \\ A University of Tasmania, School of Mathematics \& Physics, Private Bag 37, \\ Hobart, Tas 7001. Email: andrew.cole@utas.edu.au
}

Received 2009 October 1, accepted 2009 November 17

\begin{abstract}
In this review I summarise recent advances in our understanding of the importance of starburst events to the evolutionary histories of nearby galaxies. Ongoing bursts are easily diagnosed in emission-line surveys, but assessing the timing and intensity of fossil bursts requires more effort, usually demanding colormagnitude diagrams or spectroscopy of individual stars. For ages older than $\sim 1 \mathrm{Gyr}$, this type of observation is currently limited to the Local Group and its immediate surroundings. However, if the Local Volume is representative of the Universe as a whole, then studies of the age and metallicity distributions of star clusters and resolved stellar populations should give statistical clues as to the frequency and importance of bursts to the histories of galaxies in general. Based on starburst statistics in the literature and synthetic colour-magnitude diagram studies of Local Group galaxies, I attempt to distinguish between systemic starbursts that strongly impact galaxy evolution and stochastic bursts that can appear impressive but are ultimately of little significance on gigayear timescales. As a specific case, it appears as though IC 10, the only starburst galaxy in the Local Group, falls into the latter category and is not fundamentally different from other nearby dwarf irregular galaxies.
\end{abstract}

Keywords: galaxies: dwarf — galaxies: starburst

\section{Introduction}

Under Concordance Cosmologies, galaxy mergers both major and minor are expected to play a central role in the evolution of virtually every galaxy. Starbursts triggered by tidal and/or hydrodynamic interactions between galaxies are a major driver of morphological transformation and, though rare, are prominent contributors to near- and midinfrared emission in the Universe. As just one example, starbursts driven by major mergers are thought to be an important driver in the creation of giant elliptical galaxies (Renzini 2006).

Typical large galaxies generally behave as approximately self-regulated systems and exhibit a relatively smoothly varying star formation rate that depends on gas density (e.g. Kennicutt 1998a), and is likely to slowly decline with time subsequent to their initial formation (Larson \& Tinsley 1978). Starbursting states in large galaxies are rare events, likely triggered by mergers or strong tidal interactions (e.g. Mihos \& Hernquist 1996; Samland \& Gerhard 2003), and are often easily distinguished from a simple scaling-up of the global starformation rate by manifesting as highly localized nuclear or circumnuclear starbursts (e.g. Kennicutt 1998b).

Conversely, small galaxies are not expected to show constant or smoothly declining star formation rates, because they are far more susceptible to disruption by internal feedbacks and external perturbations (e.g. Stinson et al. 2007). This presents an observational challenge, because only within $10-15 \mathrm{Mpc}$ can individual stars be resolved in order to study the stellar populations of starbursts in detail, and only within $\approx 1 \mathrm{Mpc}$ can stars be resolved to ages approaching a Hubble time to characterize the underlying stellar populations and search for fossil bursts. However, within these distances luminous galaxies are rare, far outnumbered by dwarf galaxies. Thus we have plentiful opportunity to study the burstiness of small galaxies in great detail, but the significance of observed variations in SFR is complicated by the expectation of large random fluctuations intrinsic to small galaxies.

One of the challenges of the studies of dwarf galaxy star-formation histories is to distinguish between systemic starbursts that are qualitatively different from steady state or quiescent star formation, and stochastic bursts which are merely the manifestation of normal variation. Offsetting this difficulty is the advantage that in resolved stellar populations there is a large array of tools available to measure the timing, intensity, and metallicity of star-formation episodes of any age up to a Hubble time. This means that burstiness studies can be made of nearby galaxies regardless of their current morphology or gas content: even galaxies with no future have a history. The study of burst histories in early-type galaxies has the potential to illuminate the processes of hierarchical assembly of large galaxies and morphological transformation of galaxies from disk-dominated to spheroidal (e.g. Mayer et al. 2007). 
Star-forming, gas-rich galaxies are of course easier targets for study, because of the higher light-to-mass ratios of young stars and the possibility to identify optically faint systems via HI surveys. Late-type galaxies in the nearby Universe exhibit a wide range of specific star formation rates ranging from nearly inactive to extreme starburst conditions (Hunter \& Gallagher 1986). The only galaxies with neutral gas detections that do not seem to be forming stars are the lowest-luminosity examples (Hunter \& Gallagher 1985), the transition-type dwarfs such as the recently-discovered Leo T dwarf (Irwin et al. 2007). These dwarfs, which include among their number the Phoenix, Pisces, and Pegasus systems, are very faint $\left(M_{\mathrm{B}}>-14\right)$, isolated systems. They may be showing the signs of a breathing mode of star formation (Stinson et al. 2007), in effect experiencing an 'anti-starburst', or they could merely be forming stars at such a low rate that no star massive enough to ionize hydrogen has been produced within the past $\approx 10^{7}$ years (Lee et al. 2009).

In this review paper I will not discuss the characteristics and causes of ongoing strong starbursts; an excellent summary of the subject can be found in (e.g. Gallagher 2005). The purpose of this paper is to review what is known about the role of bursts over the lifetimes of nearby galaxies, to discuss the lines of evidence that could be used to infer the presence of fossil bursts in resolved stellar populations, and to draw attention to some of the recent work on starburst statistics and durations in the Local Volume. For a thorough discussion of star formation in all modes and galaxy types, including the starburst phenomenon in context, see the review by Kennicutt (1998b), and references therein.

Since the majority of the evidence for fossil starbursts must be gleaned from color-magnitude diagrams (CMDs), the most detailed results are by necessity confined to the Local Group, galaxies within about $\sim 1 \mathrm{Mpc}$ of the barycentre of the M31-Milky Way pair. Within this group of $\approx 50$ galaxies, we find examples of late-type galaxies experiencing both booms and busts in their current specific star-formation rate; of galaxies that burst during their formation and never again; of galaxies that burst multiple times at inteverals of several Gyr; and galaxies for which there is no evidence of strong variations in SFR at all. There even appears to be a galaxy which saved much its gas for 5 billion years after its first star formation, whereupon it experienced a major SFR event at a lookback time corresponding to a redshift $z \approx 1$; there are hints that this type of star-formation history (SFH) may be commonplace among the most isolated small galaxies. Comprehensive reviews of the SFH of Local Group galaxies are to be found in Mateo (1998) and Tolstoy, Hill \& Tosi (2009), among others.

\section{Dwarf Starbursts: Prevalence and Properties}

The archetypal dwarf galaxy starburst is M82 (Gallagher \& Smith 1999, and references therein). It displays most of the characteristics associated with an extreme starburst environment, including the formation of massive super star clusters, strong infrared emission, remarkably high $\mathrm{H} \alpha$ equivalent width, strong tidal interaction with a massive neighbor, and a galactic wind driven by the large number of supernovae resulting from the starburst. This is an incontrovertible example of star formation in a dramatically different mode from that experienced by most dwarfs in the nearby Universe.

Quite different, but equally important examples of starbursts in dwarf galaxies are the blue compact dwarfs (BCDs): the 'extragalactic HII regions' of Sargent \& Searle (1970). Because BCDs are a rare galaxy type, they are typically only found at distances greater than $10 \mathrm{Mpc}$, and attention naturally focuses on their highest surface brightness features. However, unlike M82, BCDs are not commonly found to be interacting with massive companions, and may but do not necessarily show evidence for the formation of massive star clusters or unusually centrally concentrated star formation (e.g. Aloisi, Tosi \& Greggio 1999). This variation of detail speaks to the probability that star formation in small galaxies is subject to stochastic fluctuations, possibly leading to strong differences in instantaneous SFR without implying a qualitative difference in the modes, triggers, and timescales of star formation (e.g. Weisz et al. 2008).

There has been a tremendous amount of work done in cataloguing the properties and populations of dwarf starbursts within $\approx 10 \mathrm{Mpc}$, but completeness and homogeneity of data have been high barriers to putting their properties into context and beginning to assess the importance of various physical processes at work. Recently, a number of surveys have come together to scale this barrier, providing deep and uniform samples of galaxy broadband luminosities and colors, $\mathrm{H} \alpha$ equivalent widths, and neutral gas content (e.g. Salzer et al. 2001; Brinchmann et al. 2004; Meurer et al. 2006; Kennicutt et al. 2008; Dalcanton et al. 2009, among others). These allow, for the first time, statistically sound estimates of the fraction of starbursting galaxies, the fraction of total star formation that occurs in bursts, and the duration of typical starbursts.

A thorough review of all the recent survey work on dwarf galaxies in the Local Volume is far beyond the scope of this paper, so I will focus on one set of recent results that bears directly on the burstiness of small galaxies. A major result of the $11 \mathrm{Mpc} \mathrm{H} \alpha$ UV Galaxy Survey (11HUGS) survey has been published by Lee et al. (2009), giving star formation rates based on $\mathrm{H} \alpha$ luminosities for over 300 galaxies within $11 \mathrm{Mpc}$, complete down to apparent magnitude $\mathrm{B} \approx 15$. This allows a complete census of star fomation rates in small galaxies, particularly attuned to studies of starburst statistics when a starburst is defined purely in terms of the ratio of current SFR to lifetime average. The major findings of Lee et al. (2009) can be summarized as follows:

- Dwarf galaxies with $\mathrm{H} \alpha$ equivalent widths $>100 \AA$ make up only $6_{-2}^{+4} \%$ of late-type galaxies, and a correspondingly low fraction of star formation, $23_{-9}^{+14} \%$, occurs during burst events. 
- Non star-forming late-type galaxies are as rare as starbursts. However, the galaxies that lack $\mathrm{H} \alpha$ emission are all fainter than $M_{\mathrm{B}}<-13.6$. Therefore sampling effects on the initial mass function mean that small amounts of star formation, in keeping with low overall galaxy masses, could be taking place without necessarily producing any star massive enough to ionize its natal cloud.

- It is likely that star formation never ceases completely between bursts, instead falling to a rate $\approx 4$ times less than the peak rate, on average. This conclusion rests on the assumption that every galaxy is equally likely to become the host of a burst; further work is needed to test this assumption.

The 11HUGS dataset sets a new standard for the statistics of star formation properties of nearby dwarf galaxies, and the conclusions of Lee et al. (2009) make a very secure foundation on which to build a comprehensive theory of star formation in small galaxies. The conclusions based on $\mathrm{H} \alpha$ data will be extended in time by the addition of ultraviolet data, owing to the fact that Balmer continuumbright B stars live for an order of magnitude longer on the main-sequence than do Lyman continuum-bright $\mathrm{O}$ stars.

Further extension to the time baseline can be provided by diffraction-limited imaging that is capable of resolving individual stars as deeply as signal-to-noise and crowding permit. It is the addition of the temporal component for studies of an individual galaxy that will ultimately provide the complete picture of the burstiness of star formation. Such data have been obtained for Local Group galaxies and for star-forming galaxies up to several Mpc away and ages of up to $10^{9} \mathrm{yr}$ (e.g. Cannon et al. 2003; McQuinn et al. 2009), with the result that the typical starburst in dwarf galaxies appears to last for a few times $10^{8}$ years. During these periods of heightened SFR, the sites of star formation move around the galaxy, propagating at speeds of $\sim 10 \mathrm{~km} \mathrm{~s}^{-1}$ (e.g. Dohm-Palmer et al. 1998) and producing what would be observable as a 'flickering' if the SFR at just one location was to be measured (McQuinn et al. 2009). These independent constraints on the duration and duty cycle of starbursts provide complementary information to the statistics of current bursts embodied by the 11HUGS results, and should be strong constraints on numerical models that attempt to account for fluctuations in SFR in small galaxies (e.g. Stinson et al. 2007).

\section{Identifying Starburst Fossils}

If strong starbursts are assumed to account for $\sim 25 \%$ of the lifetime integrated star formation in dwarf galaxies, then it should be possible to see the evidence for fossil bursts in the resolved stellar populations of nearby galaxies. The most direct way to unearth fossil bursts is via direct probes of the star formation history of a galaxy through analysis of its color-magnitude diagram (CMD, Tosi et al. 1991; Tolstoy \& Saha 1996; Dolphin 2002). As a stellar population ages, the absolute magnitude of its main-sequence turnoff increases, enabling direct tests of stellar mass contained in a galaxy as a function of age. The classical approach to this problem is to overlay theoretical stellar isochrones of various age and metallicity combinations on the observed CMD and thereby identify the characteristics of the dominant stellar populations. Particularly narrow sequences similar to star cluster CMDs would be indicative of a burst of star formation, while gaps in the CMD result from quiescent epochs in the life of the galaxy.

For most galaxies the process cannot simplified to such an extent, beacuse of the continuous distribution of stellar ages and metallicities, and the resulting extremely large number of different isochrone combinations to be tested. Quantitative estimates of stellar masses formed are made difficult in composite populations (i.e. nearly every galaxy in the Universe), because older populations are masked to some extent by the low-mass members of younger populations, and metallicity evolution can counteract some of the dimming effects of age. The solution to this problem is to compare the observed density of stars in a CMD to probability distributions formed by the convolution of isochrones with an initial mass function. The difference between the synthetic CMDs thus created and a given dataset can then be minimized using a nonlinear least-squares approach. Other properties of the stellar populations, including the dispersion in interstellar reddening values, proportion of binary stars, and detailed elemental abundance ratios (e.g. $[\alpha / \mathrm{Fe}]$ ) may also require modelling in order to obtain meaningful results. The coefficients returned by the minimization procedure correspond to the star-formation rate as a function of time (and metallicity, reddening, $[\alpha / \mathrm{Fe}]$, or any other parameters the investigator thinks their data can constrain).

Such techniques of CMD fitting have been widely applied throughout the Local Group over the past 2 decades, and the results have been reviewed comprehensively by Mateo (1998) and Tolstoy, Hill \& Tosi (2009), among others. The field was brought to maturity by the diffraction-limited imaging of the Hubble Space Telescope, which overcomes much of the stellar crowding that plagued ground-based imaging. It has been possible with HST to directly measure the SFH of galaxies over their entire history, with a time resolution of about $10 \%$ of the age, throughout the Local Group. Observations of more distant galaxies have been limited to younger lookback times by the practical limit of HST imaging at magnitude $\approx 29-30$ for most projects. This means that for some of the most extreme and interesting starbursts, e.g. M82 $\left(d \approx 3.9 \mathrm{Mpc}, m-M_{0} \approx 27.9\right)$ direct age-dating via the main-sequence turnoff is not possible for ages greater than a few times $10^{9}$ years.

\subsection{Indirect Probes of Burst Histories}

Current starbursts are visible to great distances, but fossil bursts begin to fade and rapidly become difficult to accurately characterize. No better example of the blurring effects of time is available than the case of the Large and Small Magellanic Clouds. At a distances of just 48 (LMC) 
and 55 (SMC) kpc from the Milky Way, and with a centerto-center separation of $22 \mathrm{kpc}$, the Magellanic Clouds are obviously and strongly interacting with the Milky Way and with each other. However, neither galaxy is currently experiencing a starburst, and there has been great debate in the literature over the degree of burstiness in their past histories. It is far beyond the scope of this review to discuss all of the observational evidence pro and con for a bursting SFH as opposed to a smooth SFH, but the arguments can be followed in the proceedings of IAU Symposia devoted to the Magellanic system (Chu et al. 1999; van Loon \& Oliveira 2009), the monograph by Westerlund (1997), and the excellent review by Olszewski, Suntzeff \& Mateo (1996).

In short, the LMC contains several globular clusters like those in the Milky Way, plus a large number of younger, massive, dense clusters unlike any found in the Galaxy. These young globulars have age distributions peaked at ages of $\approx 100-200 \mathrm{Myr}$ and $\approx 1-2 \mathrm{Gyr}$, leading to suggestions that the LMC must have experienced strong starbursts at those ages. The SMC also contains young, massive clusters, and their ages also appear to be concentrated at specific times (Rich et al. 2000), although not at the same times as the LMC. CMD studies of field stars in the LMC based on HST imaging found varying degrees of evidence for a bursting SFH, but it became apparent that the LMC never completely ceased forming stars in the periods between its epochs of prolific cluster formation, and these data were used to argue in favor of a SFH that was more smooth than bursty.

The most complete derivations of the SFH of the Clouds are to be found in the work of Harris \& Zaritsky (2004, SMC) and Harris \& Zaritsky (2009, LMC), and the results show that the star formation activity in the field was indeed peaked during the times of massive cluster formation, and the times of activity correlate between the two Clouds. It therefore does appear that the production of massive star clusters is a signature of major events in the life of a galaxy, indicative of star formation under different conditions than normal, 'quiescent' star formation. Following the example of the Magellanic Clouds, the presence of massive star clusters can be a tracer of fossil starbursts when the evidence from field star ages is insufficient to unambiguously identify a burst.

The production of massive star clusters is not expected as the result of the normal, stochastic burstiness one sees in dwarf galaxies. The presence of massive clusters can be diagnostic of bona fide, systemic starbursts, and if the clusters are dense enough to escape dissolution in the tidal field of the galaxy, they can be powerful probes over a long time baseline. This is particularly useful because massive clusters are far more easily observable than even the brightest single supergiant stars, and can thus trace starbursts to larger distances and more crowded environments (e.g. Gallagher \& Smith 1999). Super star clusters are indeed a ubiquitous feature of starburst galaxies, and in some starbursts the clusters appear likely to survive to high ages and eventually appear similar to globular clusters (e.g. de Grijs, O’Connell \& Gallagher 2001, and references therein).

The burstiness of star formation can also have strong implications for the chemical enrichment of galaxies (e.g. Kobulnicky \& Skillman 1996, 1997), and these chemical signatures persist in the subsequent generations of stars, long after the bursts responsible have faded away. Starbursts can rapidly enrich the interstellar medium of a galaxy, which might otherwise be diluted in metal abundance by the infall of fresh metal-poor gas during 'quiescent' star formation. The timescales of starbursts can potentially be probed by the measuring the abundance ratios of elements produced in Type II and Type Ia supernovae, and in AGB stars (Tolstoy, Hill \& Tosi 2009, and references therein). However, if the starburst is extreme enough to produce a large number of supernovae in a small galaxy, then the metals produced could be lost to the intergalactic medium rather than incorporated into subsequent stellar generations, reducing the effective metal yield of the population in a starburst (Mac Low \& Ferrara 1999; Lee et al. 2006). The idea of identifying the star-formation environments of stars by identifying telltale patterns of chemical enrichment has enormous potential for understanding the formation and evolution of galaxies from the earliest times to the present day; for a detailed description of the promise and challenge of this approach, see the review by Freeman \& Bland-Hawthorn (2002).

\section{Local Group Case Studies}

\subsection{The Carina Dwarf Spheroidal}

The dwarf spheroidal galaxies are low-luminosity $\left(M_{\mathrm{B}}>-14\right)$, late-type galaxies that are nearly exclusively observed as satellites of the Milky Way (or M31) with distances of $25 \leq r_{\mathrm{gc}} \leq 250 \mathrm{kpc}$ (Mateo 1998). Many of them appear to have only ever experienced one episode of star formation, at the earliest epochs, leading to much conjecture about the mechanisms for their gas-loss and their survival (see the discussions in Tolstoy, Hill \& Tosi (2009) and Mateo (1998) for further information). Especially among the lower-luminosity and less distant spheroidals, the star formation was restricted to more or less old ages (e.g. Mateo et al. 1991; Dolphin 2002), although some chemical evolution has been reported (e.g. Tolstoy et al. 2004), indicating some complexity to the SFH. The most luminous dwarf spheroidals, the Fornax and Sagittarius systems, show both extended SFHs and small globular cluster populations.

Despite the overall trends pointing to old populations and simple SFHs, the classic example of a galaxy with a history of repeated burst cycles is a dwarf spheroidal, the Carina system. At a distance of $100 \mathrm{kpc}$, Carina has been known to harbor a large intermediate-age population since the work of Mould \& Aaronson (1983). The first imaging to reach the level of the horizontal branch and heliumburning red clump revealed a surprisingly bimodal stellar distribution implying a bursting or gasping SFH (SmeckerHane et al. 1994). Subsequent deeper imaging confirmed 
this and revealed that the dominant stellar population was formed in a burst about 7-9 Gyr ago, with clearly separated bursts at both older $(>11 \mathrm{Gyr})$ and younger ( $\approx 4$ Gyr) ages (Hurley-Keller, Mateo \& Nemec 1998). Medium-resolution spectroscopy of individual red giant stars revealed that the metallicity of the younger stars is on average slightly higher than that of the older stars, demonstrating chemical evolution with time (Koch et al. 2006).

Carina remains the clearest example of a galaxy in which ancient and intermediate-age bursts are so distinctly separated from each other that the quiescent epochs produce gaps in the subgiant region of the CMD. Qualitatively similar behavior is seen in several other early- and late-type dwarfs, e.g. Leo I (Dolphin 2002) and IC 1613 (Skillman et al. 2003), but the variations in SFR on Gyr timescales appear to be milder than in Carina. There are several ways in which to interpret this information. It is possible that Carina is just far enough from the Milky Way to have escaped the early stripping of gas that terminated the star formation of the closer-in spheroidals like Sextans, Ursa Minor, or Draco (Tolstoy, Hill \& Tosi 2009). Alternatively, perhaps Carina did consume or eject its entire gas content after an initial burst, but accreted more gas several gigayears later. In comparing Carina to more distant galaxies, it must be noted that some of the apparent smoothness in SFH at old ages for galaxies more than a few hundred kiloparsecs distant may yet be attributable to observational difficulties rather than genuine constancy of SFR. Because the fractional age resolution of the current generation of CMD studies of Local Group dwarfs is at best about $10 \%$, starbursts of the durations reported by, e.g., McQuinn et al. (2009) would be unresolved for ages greater than $\sim 10^{9.5} \mathrm{yr}$, causing galaxy SFHs to appear to decrease in burstiness with age.

\subsection{A Late-Bursting Galaxy: Leo A}

Carina and the other early-type, gas-free, dwarf spheroidal systems are nearly all satellites of the Milky Way, which is expected to have had a strong impact on their evolution (e.g. Kravtsov, Gnedin \& Klypin 2004). It is therefore of interest to study more distant galaxies to similar photometric depth, in order to gauge the evolution of small galaxies in relative isolation. Leo A (DDO 69) is one of the most isolated galaxies in the Local Group (Mateo 1998), $800 \mathrm{kpc}$ from the Milky Way and $1200 \mathrm{kpc}$ from M31. It was suggested by Tolstoy et al. (1998) that Leo A had formed the vast majority of its stars within the past few Gyr, making it the most likely candidate yet to be a genuinely young galaxy. This candidacy was quashed when Dolphin et al. (2002) discovered a small number of RR Lyrae type variables in Leo A, proving the presence of ancient (age $>10$ Gyr) stars. However, deep HST imaging by Cole et al. (2007) showed conclusively that Leo A formed $90 \%$ of its stellar mass more recently than $\approx 7 \mathrm{Gyr}$ ago, corresponding to a redshift of $z \approx 1$. The SFR appeared to peak between 1 and $3 \mathrm{Gyr}$ ago and then declined, with a second episode of star formation a few hundred Myr ago. This made Leo A unique among known galaxies in having such a remarkably high fraction of stars younger than $10 \mathrm{Gyr}$. In the SFH derived by Cole et al. (2007), the average SFR of Leo A from 2 to 5 Gyr ago was 4-5 times the previous long-term average SFR. If this star formation was concentrated into episodes a few hundred Myr in duration, the actual instantaneous SFR would have qualified Leo A as an extreme dwarf starburst.

It is a puzzle how such a small galaxy $\left(M_{\text {dyn }} \approx 2 \times\right.$ $10^{8} \mathrm{M}_{\odot}$ ) could have retained so much of its gas without forming significant amounts of stars for over $5 \mathrm{Gyr}$ after it first formed. This may be an example of an HI reservoir at low metallicity, with correspondingly long cooling timescale, kept in such isolation that no perturbations or fluctuations triggered star formation for many gigayears. Based on Leo A's very small radial velocity of $-18 \mathrm{~km} \mathrm{~s}^{-1}$ with respect to the Milky Way, it seems likely that the galaxy has never been in close proximity to either M31 or the Milky Way. Interestingly, similar hints of a large fraction of delayed star formation are seen in IC 1613 (Skillman et al. 2003) and DDO 210 (McConnachie et al. 2006), although not to the same extent as in Leo A. It may become apparent with further observational effort that delayed star formation with a late burst of star formation perhaps triggered by the turnaround and infall of the isolated galaxy into an intragroup medium (McConnachie et al. 2007) is a typical characteristic of the most isolated galaxies.

\subsection{Boom or Bust? The Case of IC 10}

The gas-rich dwarf $\left(M_{\mathrm{B}}=-15.6\right)$ IC 10 has long been considered the only starburst galaxy in the Local Group and thought of as something of an anomaly (e.g. Hunter 2001). IC 10 has an unusually high surface brightness for a galaxy of its size, and unusually high numbers of WolfRayet stars (Massey \& Johnson 1998), owing to its high current SFR of $\approx 0.03 \mathrm{M}_{\odot} \mathrm{yr}^{-1} \mathrm{kpc}^{-2}$ (Hunter 2001). At a distance of $\approx 800 \mathrm{kpc}$ from the Milky Way (and a mere $250 \mathrm{kpc}$ from M31), IC 10 is clearly an outlier among the dwarf irregular galaxies of the Local Group, but how much of an outlier is still a matter of debate. It sits deep in the Zone of Avoidance $\left(\ell=119^{\circ}, b=-3^{\circ}\right)$, so detailed study has been hindered by large and variable foreground reddening compounded with a significant amount of reddening internal to the dwarf, making it the last star-forming Local Group galaxy within $1 \mathrm{Mpc}$ to be imaged down to the depth of the horizontal branch (Sanna et al. 2009). Attention has naturally been focused on the rich array of young stars around the major HII complexes, leading to classification as a dwarf starburst or blue compact dwarf (Richer et al. 2001). However, the demonstration of Hunter \& Gallagher (1986) that the classification of starforming dwarfs can be strongly distance-dependent leads us to reëxamine IC 10's status as a Local Group enigma.

We have imaged a central region of IC 10, avoiding the actively starbursting area, with the Advanced Camera for Surveys (ACS) on HST, using the ACS equivalents of $\mathrm{V}$ and I filters to produce CMDs of sufficient depth to determine the SFH with timing precision of better than 
$10 \%$ to ages of $\approx 2000 \mathrm{Myr}$ and $\sim 30 \%$ over the entire lifetime of the galaxy (Cole et al. in preparation). In order to better reveal possible previous bursts of star formation and the older stellar populations in general we purposely avoided the regions previously imaged with HST (Hunter 2001). The resulting rich dataset contains a wealth of information about IC 10, as is typical for HST images of nearby dwarfs (e.g. Dalcanton et al. 2009; Tolstoy, Hill \& Tosi 2009). Of particular interest, we have identified a window in the main body of IC 10 that is virtually free of internal reddening as identified by the narrow color range of red giants and confirmed by the low HI column density at that location (Wilcots \& Miller 1998).

The density-scaled CMD (Hess diagram) of 56000 stars in this low-reddening window is shown in Figure 1 , where the magnitudes have been corrected for a distance modulus of $(m-M)_{0}=24.5$ and a reddening of $E(B-V)=0.81 \mathrm{mag}$. Isochrones from Marigo et al. (2008) have been overlaid on the Hess diagram to show the locus of stars aged 400 and $2200 \mathrm{Myr}$ with metallicity $Z=0.004([\mathrm{M} / \mathrm{H}] \approx 1 / 5$ th solar $(\mathrm{H}$. Lee et al. 2003)). This location in the galaxy is far from any sites of active star formation, so the SFR for ages less than $\approx 1 \mathrm{Gyr}$ is not expected to be representative of the galaxy as a whole. For ages older than this, the stellar velocity dispersion will have mixed stars throughout the body of the galaxy, so the SFH should be fairly typical of any random spot within the central portion of IC 10. The stellar population appears to be predominantly of intermediate age, with a strong contribution from stars a few Gyr old.

The SFH derived from this Hess diagram is shown in Figure 2. The lack of bright main-sequence stars in this specific field is reflected in the low SFR over the past $\approx 400 \mathrm{Myr}$. However, this apparently quiescent field far from the current center of starburst activity experienced its own peaks in SFR at ages of approximately 700-800 Myr and 1.5-2.5 Gyr. During both time periods, the specific SFR at this location was higher than the current galaxywide specific SFR of $0.03 \mathrm{M}_{\odot} \mathrm{yr}^{-1} \mathrm{kpc}^{-2}$ (Hunter 2001), marked with a star at $10 \mathrm{Myr}$ and extended for reference back to $14 \mathrm{Gyr}$ by the dashed line. The Hess diagram shows that the age resolution will be severely degraded for ages older than $\approx 3-4 \mathrm{Gyr}$, because the main-sequence turnoff is too faint to be well-sampled by these data. However, it is apparent that the long-term average SFR of IC 10 may have approached or exceeded its current value for much of its early history. This implies that while IC 10's starburst is visually spectacular and prolific in production of very massive stars, it is not likely to significantly increase the stellar mass of IC 10 unless it increases in intensity or lasts for longer than a few hundred Myr. One caveat is that the current specific SFR from Hunter (2001) is averaged over the $\mathrm{D}_{25}$ diameter of the galaxy, an area $\approx 23$ times the size of our low-reddening window, but the starburst activity is strongly concentrated into the high-surface-brightness HII region complexes. The peak current SFR, measured over a comparable area to that shown here, would thus be much higher than the $\mathrm{D}_{25}$-averaged values. Caution is warranted

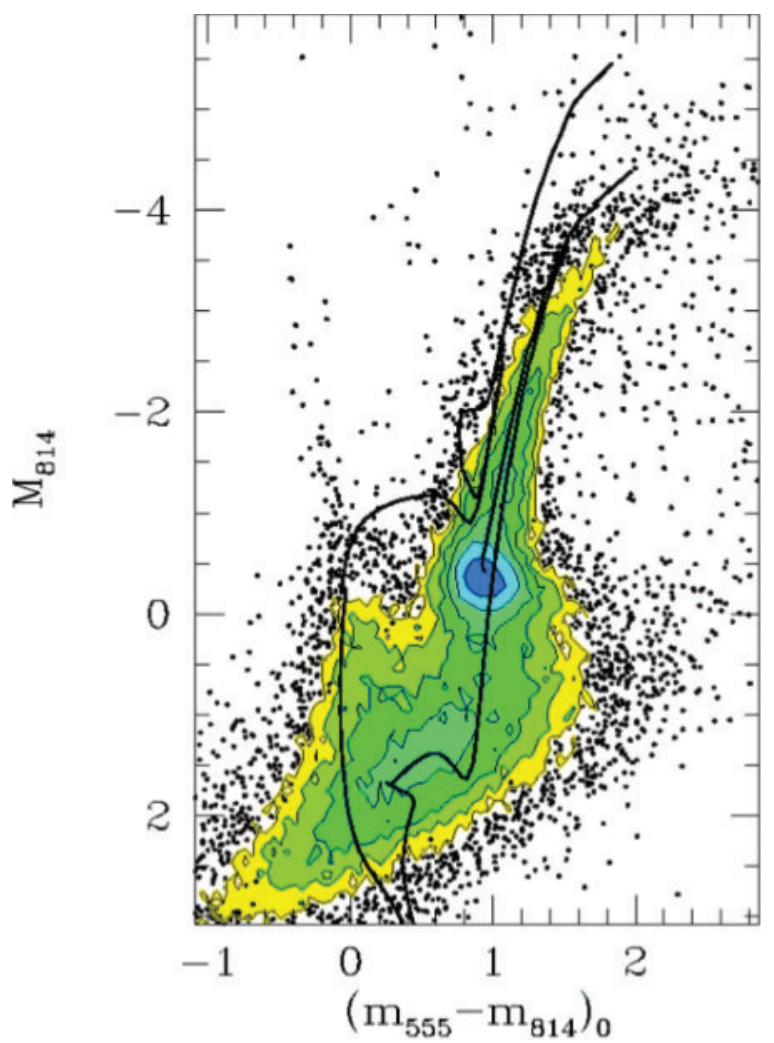

Figure 1 A color-magnitude density diagram of a low-reddening window in the northern disk of IC 10. The negligible internal reddening permits a reliable reconstruction of the SFH of the central regions of IC 10. The filters 814 and 555 are the ACS equivalents of I and V. The CMD is remarkably similar to that of NGC 6822 (Wyder 2001). Isochrones are from the Padua group, computed for a metal abundance $Z=0.004$, with ages $400 \mathrm{Myr}$ (upper track) and $2200 \mathrm{Myr}$ (lower track). $5.6 \times 10^{4}$ stars are measured in this $0.5-\operatorname{arcmin}^{2}$ window; the contours are spaced by factors of 2 in density.

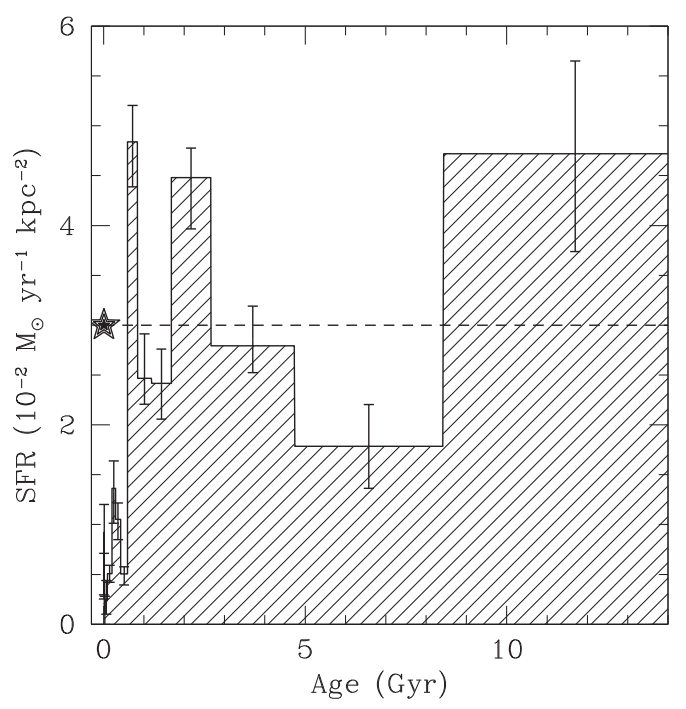

Figure 2 The SFH of IC 10 determined in our low-reddening window. For ages older than $\sim 1000 \mathrm{Myr}$ orbital motions should have mixed IC 10's populations, so the SFH should be representative of the galaxy as a whole for intermediate and old ages. Note that by the standards of its own historical average, IC10's current SFR (star and dashed line, Hunter 2001) is not abnormal. 
in making comparisons of this sort between studies of widely disparate areas. However, the finite velocity dispersion of stellar populations coupled with the decreasing in time resolution of the CMD with age perform a sort of natural averaging on the resolved stellar data, and this comparison should be valid for the older ages considered, provided we have not had the misfortune to study a 'special' location in the galaxy where star formation has been unusually enhanced or suppressed on Gyr timescales.

IC 10 has roughly the same stellar mass, dynamical mass, and average stellar age as the prototypical dwarf irregular NGC 6822, the isolated dwarf irregular WLM, and the Milky Way companion Small Magellanic Cloud. In integrated properties, there is little to distinguish between the four galaxies (H. Lee et al. 2003; Demers, Battinelli \& Letarte 2004; Orban et al. 2008; Cole et al. in preparation). The similarity to NGC 6822 goes even farther, as both galaxies are in the midst of a minor merger or neutral gas accretion event (Hunter 1997; Wilcots \& Miller 1998; de Blok \& Walter 2006). The SFH for NGC 6822 determined by Wyder (2001) even bears similarity to the long-term average SFH for IC 10 presented in Figure 2, characterized by roughly contant levels with evidence for a recent decline - punctuated by enhancements or bursts that may be connected to the presence of infalling gas. The presence of dense star clusters in NGC 6822 (e.g. Wyder 2001), which are lacking in IC 10 (Hunter 2001; Cole et al. in preparation) suggests that if anything, IC 10 has had the less bursty SFH of the two. It seems possible that if we viewed IC 10 from a vantage point in M31 instead of through the plane of the Milky Way, we would think of IC 10 as a prototype dwarf irregular, with NGC 6822 as a distant analogue.

\section{Conclusions}

The burstiness of galaxies is a fundamental observable clue to understanding the physics of star formation and the processes which drive galaxy evolution. Starbursts, galaxies that are forming stars at such a high rate that the background light of all previously formed stars pales to insignifcance (Sandage 1963), are the extreme star formation environment in the Universe. Starbursts were likely the dominant mode of star formation at high redshift (Dressler et al. 2009, and references therein), but are not 'steady state' phenomena in any sense (Rieke \& Low 1975), and their prevalence is low among luminous galaxies in the nearby Universe, often tied to mergers and interactions (e.g. Kennicutt 1998b).

Late-type galaxies in the nearby Universe exhibit a wide range of specific star formation rates ranging from nearly inactive to extreme starburst conditions (Hunter \& Gallagher 1986; Lee et al. 2009). This is not surprising, as on theoretical grounds it is expected that galaxies with dynamical masses $M_{\text {tot }}<\sim 10^{9.5} \mathrm{M}_{\odot}$ become unstable to their own stellar feedback from winds and supernovae (e.g. Mac Low \& Ferrara 1999; Stinson et al. 2007, and references therein). For small galaxies, this makes the dichotomy between 'starbursting' and 'quiescent' states somewhat artificial, because the natural state of dwarf galaxies is to show a continuum of burstiness (Weisz et al. 2008) related to factors both internal (dynamical mass, angular momentum profile, gas content) and external (tidal interactions, ionizing background). If a starburst is defined by a stellar birthrate that exceeds $2-3$ times the long-term average, then dwarf galaxies are much more susceptible to experiencing bursty star formation histories than are giant galaxies. While large galaxies most likely require disruptive events and extreme conditions in order to experience a starburst, dwarf galaxies should be able to meet the observational definition of a starburst without qualitatively changing the mode of star formation, i.e. by producing large numbers of massive clusters, concentrating the star formation to the circumnuclear region of the galaxy, or experiencing major reorganizations of gas content or morphology.

Within $10 \mathrm{Mpc}$, star formation 'booms' are rare, with only $6 \%$ of dwarf galaxies showing a current SFR more than 2.5 times their long term average (Lee et al. 2009). However, 'busts' are equally rare, indicating that as long as neutral gas is present, some star formation occurs. In general, the early conclusion of Hunter \& Gallagher (1985) suggesting that star formation in dwarf irregulars is 'down but not out' has been borne out and put on a firm statistical footing by subsequent work. Most recent work, typified by Lee et al. (2009), suggests that between 20 and $30 \%$ of star formation occurs during burst episodes. These episodes seem to last, very roughly, $\sim 10^{8.5} \mathrm{yr}$ (e.g. McQuinn et al. 2009). Note that these figures do not distinguish between systemic bursts, which are not sustainable over the long term and may be signifcant in their production of star clusters and metals or for their promotion of morphological transformation, and stochastic bursts of the kind which are predicted by numerical models (e.g. Stinson et al. 2007) and observed in nearby dwarfs (e.g. Weisz et al. 2008). It is interesting to note that while models predict a high degree of burstiness, increasing with decreasing galaxy mass, CMD analysis indicates relatively smooth SFHs for most Local Group galaxies. Either some unmodelled factor is acting to suppress the burstiness seen in the models, or the CMD analyses are less sensitive than predicted to factors of 2 variation in SFR over time periods of a few hundred Myr.

The isolated dwarf galaxy Leo A appears to have waited several Gyr before forming the vast majority of its stars in an event that itself spanned several Gyr. An upper limit of $\approx 10 \%$ on the fraction of stars older than $8 \mathrm{Gyr}$ was found by Cole et al. (2007). This makes it unique among galaxies that have been studied to the depth of the oldest main-sequence turnoff with the Hubble Space Telescope, but hints of similar behavior may be visible in other isolated galaxies (e.g. McConnachie et al. 2006). If the delayed burst is a natural feature of dwarf galaxies, then it has not been captured by the models that predict steady 'breathing' pulses of star formation. One possibility is that cosmic reionization heated but did not evaporate the neutral gas from the potential well of the galaxy 
and long cooling times produced the delay (e.g. Bullock, Kravtsov \& Weinberg 2000). On the other hand, if the late-blooming burst was triggered by a merger or accretion event, there is no hope at this late time, several Gyr later, of identifying the trigger.

The galaxy IC 10 appears to have much in common with similar size dwarf irregulars and is not currently forming stars far above its long-term average rate. While it has repeatedly been referred to as the nearest starburst galaxy and an anomaly within the Local Group, the star formation history derived from HST imaging places it squarely within a continuum of similar-mass late-type galaxies, bearing a strong family resemblance to archetypal irregulars NGC 6822 and the Small Magellanic Cloud. However the burst age is only a few times $10^{7} \mathrm{yr}$, indicating that the burst may be at a very early stage if IC 10 has similar properties to other dwarf starbursts, which appear to last for $\sim 10$ times longer (e.g. McQuinn et al. 2009). IC 10 is similar in mass and metallicity to the SMC, but has failed to produce any massive star clusters during its lifetime, indirect evidence that the SMC has had the more tumultuous history and experienced a more intense mode of star formation at past epochs.

\section{Acknowledgments}

This article is a summary of a review talk presented at the Southern Cross Astrophysics Conference, on the subject of 'Galaxy Metabolism', in June 2009. The author gratefully acknowledges the conference organisers for travel support, and the conference participants for lively and informative discussion that influenced the form and content of this article.

\section{References}

Aloisi, A., Tosi, M. \& Greggio, L., 1999, AJ, 118, 302

Brinchmann, J. et al., 2004, MNRAS, 351, 1151

Bullock, J. S., Kravtsov, A. V. \& Weinberg, D. H., 2000, ApJ, 539, 517 Cannon, J. M., Dohm-Palmer, R. C., Skillman, E. D., Bomans, D. J., Côté, S. \& Miller, B. W., 2003, AJ, 126, 2806

Chu, Y.-H., Suntzeff, N., Hesser, J. \& Bohlender, D., 1999, New Views of the Magellanic Clouds, IAUS 190 (San Francisco: ASP) Cole, A. A. et al., 2007, ApJ, 659, L17

Dalcanton, J. J. et al., 2009, ApJS, 183, 67

de Blok, W. J. G. \& Walter, F., 2006, AJ, 131, 343

de Grijs, R., O’Connell, R. W. \& Gallagher, J. S., III, 2001, AJ, 121,768

Demers, S., Battinelli, P. \& Letarte, B., 2004, A\&A, 424, 125

Dolphin, A. E., 2002, MNRAS, 332, 91

Dolphin, A. E. et al., 2002, AJ, 123, 3154

Dohm-Palmer, R. C. et al., 1998, AJ, 116, 1227

Dressler, A., Oemler, A., Gladders, M. G., Bai, L., Rigby, J. R. \& Poggianti, B. M., 2009, ApJ, 699, L130

Freeman, K. C. \& Bland-Hawthorn, J., 2002, ARA\&A, 40, 487

Gallagher, J. S., III, 2005, Ap\&SSL, 329, 11

Gallagher, J. S., III \& Smith, L. J., 1999, MNRAS, 304, 540

Harris, J. \& Zaritsky, D., 2004, AJ, 127, 1531

Harris, J. \& Zaritsky, D., 2009, AJ, 138, 1243

Hunter, D. A., 1997, PASP, 109, 937

Hunter, D. A., 2001, ApJ, 559, 225

Hunter, D. A. \& Gallagher, J. S., III, 1985, ApJS, 58, 533

Hunter, D. A. \& Gallagher, J. S., III, 1986, PASP, 98, 5

Hurley-Keller, D., Mateo, M. \& Nemec, J., 1998, AJ, 115, 1840
Irwin, M. J., 2007, ApJ, 656, L13

Kennicutt, R. C., 1998a, ApJ, 498, 541

Kennicutt, R. C., 1998b, ARA\&A, 36, 189

Kennicutt, R. C., Lee, J. C., Funes, J. G., Sakai, S. \& Akiyama, S., 2008, ApJS, 178, 247

Kobulnicky, H. A. \& Skillman, E. D., 1996, ApJ, 471, 211

Koch, A., Grebel, E. K., Wyse, R. F. G., Kleyna, J. T., Wilkinson, M. I., Harbeck, D. R., Gilmore, G. F. \& Evans, N. W., 2006, AJ, 131,895

Kravtsov, A. V., Gnedin, O. Y. \& Klypin, A. V., 2004, ApJ, 609, 482

Larson, R. B. \& Tinsley, B. M., 1978, ApJ, 219, 46

Lee, H., McCall, M. L., Kingsburgh, R. L., Rossi, R. \& Stevenson, C. C., 2003, AJ, 125, 146

Lee, H., Skillman, E. D., Cannon, J. M., Jackson, D. C., Gehrz, R. D., Polomski, E. F. \& Woodward, C. E., 2006, ApJ, 647, 970

Lee, J. C., Kennicutt, R. C., Jr, Funes, J. G., Sakai, S. \& Akiyama, S., 2009, ApJ, 692, 1305

Mac Low, M.-M. \& Ferrara, A., 1999, ApJ, 513, 142

Marigo, P., Girardi, L., Bressan, A., Groenewegen, M. A. T., Silva, L. \& Granato, G. L., 2008, A\&A, 482, 883

Massey, P. \& Johnson, O., 1998, ApJ, 505, 793

Mateo, M., 1998, ARA\&A, 46, 435

Mateo, M., Nemec, J., Irwin, M. \& McMahon, R., 1991, AJ, 101, 892

Mayer, L., Kazantzidis, S., Mastropietro, C. \& Wadsley, J., 2007, Natur, 445, 738

McConnachie, A. W., Arimoto, N., Irwin, M. \& Tolstoy, E., 2006, MNRAS, 373, 715

McConnachie, A. W., Venn, K. A., Irwin, M. J., Young, L. M. \& Geehan, J. J., 2007, ApJ, 671, L33

McQuinn, K. B., Skillman, E. D., Cannon, J. M., Dalcanton, J., Dolphin, A., Stark, D. \& Weisz, D., 2009, ApJ, 695, 561

Meurer, G. et al., 2006, ApJS, 165, 307

Mihos, J. C. \& Hernquist, L., 1996, ApJ, 464, 641

Mould, J. \& Aaronson, M., 1983, ApJ, 273, 530

Olszewski, E. W., Suntzeff, N. B. \& Mateo, M., 1996, ARA\&A, 34,511

Orban, C., Gnedin, O. Y., Weisz, D. R., Skillman, E. D., Dolphin, A. E. \& Holtzman, J. A., 2008, ApJ, 686, 1030

Renzini, A., 2006, ARA\&A, 44, 141

Rich, R. M., Shara, M., Fall, S. M. \& Zurek, D., 2000, AJ, 119, 197

Richer, M. G. et al., 2001, A\&A, 370, 34

Rieke, G. H. \& Low, F. J., 1975, ApJ, 197, 17

Salzer, J. J. et al., 2001, AJ, 121, 66

Samland, M. \& Gerhard, O. E., 2003, A\&A, 399, 961

Sandage, A., 1963, ApJ, 138, 863

Sanna, N. et al., 2009, ApJ, 699, L84

Sargent, W. L. W. \& Searle, L., 1970, ApJL, 162, L155

Sillman, E. D., Tolstoy, E., Cole, A. A., Dolphin, A. E., Saha, A., Gallagher, J. S., Dohm-Palmer, R. C. \& Mateo, M., 2003, ApJ, 596,253

Smecker-Hane, T. A., Stetson, P. B., Hesser, J. E. \& Lehnert, M. D., 1994, AJ, 108, 507

Stinson, G. S., Dalcanton, J. J., Quinn, T., Kaufmann, T. \& Wadsley, J., 2007, ApJ, 667, 170

Tolstoy, E. \& Saha, A., 1996, ApJ, 462, 672

Tolstoy, E., Hill, V. \& Tosi, M., 2009, ARA\&A, 47, 371

Tolstoy, E. et al., 1998, AJ, 116, 1244

Tolstoy, E. et al., 2004, ApJ, 617, L119

Tosi, M., Greggio, L., Marconi, G. \& Focardi, P., 1991, AJ, 102, 951 van Loon, J. Th. \& Oliveira, J. M., 2009, The Magellanic System: Stars, Gas and Galaxies, IAUS 256 (Cambridge: Cambridge University Press)

Weisz, D. R., Skillman, E. D., Cannon, J. M., Dolphin, A. E., Kennicutt, R. C., Jr, Lee, J. C. \& Walter, F., 2008, ApJ, 689, 160

Westerlund, B. E., 1997, The Magellanic Clouds (Cambridge: Cambridge University Press)

Wilcots, E. M. \& Miller, B. W., 1998, AJ, 116, 2363

Wyder, T. K., 2001, AJ, 122, 2490 